\title{
Selective Vulnerability of the Medullary Thick Ascending Limb to Anoxia in the Isolated Perfused Rat Kidney
}

\author{
Mayer Brezis, Seymour Rosen, Patricio Silva, \\ and Franklin H. Epstein \\ Charles A. Dana Research Institute, Harvard-Thorndike \\ Laboratory of Beth Israel Hospital, Departments of Medicine and \\ Pathology, Harvard Medical School and Beth Israel Hospital, \\ Boston, Massachusetts 02215
}

bstract. A specific anatomical lesion sharply localized to the cells of the medullary thick ascending limbs (mTAL) and characterized by mitochondrial swelling progressing to nuclear pyknosis and cell death is elicited reproducibly in isolated rat kidneys perfused for 15 or 90 min with cell-free albumin-Ringer's medium gassed with $5 \% \mathrm{CO}_{2}, 95 \% \mathrm{O}_{2}\left(\mathrm{O}_{2}\right.$ content, $\left.1.5 \mathrm{vol} / 100 \mathrm{ml}\right)$. The lesion, involving about half of mTALs, appears first in mTALs removed from vascular bundles and near the inner medulla, areas most likely to be anoxic. Hypoxic perfusion $\left(\mathrm{O}_{2}\right.$ content $\left.0.12 \mathrm{vol} / 100 \mathrm{ml}\right)$ exaggerates the lesion, wiping out gradations of damage and extending it to all mTALs. $\mathrm{O}_{2}$-enriched perfusions using rat erythrocytes $\left(\mathrm{O}_{2}\right.$ content $\left.7.1 \mathrm{vol} / 100 \mathrm{ml}\right)$ completely eliminates the lesion (unless gassed with carbon monoxide). Similarly, supplementation of the perfusion medium with a purified hemoglobin $\left(\mathrm{O}_{2}\right.$ content $\left.5.8 \mathrm{vol} / 100 \mathrm{ml}\right)$ prevents mTAL injury. Perfusion with a fluorinated hydrocarbon blood substitute, Oxypherol $\left(\mathrm{O}_{2}\right.$ content $\left.4.3 \mathrm{vol} / 100 \mathrm{ml}\right)$ also attenuates the lesion.

These findings suggest that the mTAL is exquisitely susceptible to anoxic damage because of low $\mathrm{O}_{2}$ supply imposed by the medullary vascular system and the high rate of metabolism mandated by active reabsorption of sodium chloride. The vulnerability of the mTAL to anoxic injury could play a key role in the pathogenesis of ischemic renal injury.

Dr. Brezis is a recipient of a fellowship from the American Physicians Fellowship for Israel.

Received for publication 14 February 1983 and in revised form 26 September 1983.

J. Clin. Invest.

(c) The American Society for Clinical Investigation, Inc. 0021-9738/84/01/0182/09 \$1.00

Volume 73 , January $1984,182-190$

\section{Introduction}

During isolated perfusion of the rat kidney, a specific lesion is consistently observed, occurring within $15 \mathrm{~min}$, confined to the medullary thick ascending limb (mTAL) ${ }^{1}$ of Henle's loop, which progresses from mitochondrial swelling to nuclear pyknosis and complete cellular disruption (1). Failure of urine-concentrating ability, defective distal sodium reabsorption with reduced glomerular filtration rate (GFR) and negative tubuloglomerular feedback, and increased potassium excretion all appear to be functional consequences of this injury (2). The addition of amino acids to the perfusate has been shown to attenuate the anatomical damage and to improve kidney function (2), but the basis for the mTAL lesion has remained obscure.

Recent observations by Epstein et al. (3) using organ spectrophotometry in whole isolated perfused kidneys, have indicated that a significant fraction (20-40\%) of cytochrome $a, a_{3}$ (cytochrome $c$ oxidase) as sensed by this technique is reduced but becomes more oxidized when mTAL transport is decreased by furosemide (3). Cytochrome $a, a_{3}$ is normally $95-98 \%$ oxidized in the presence of oxygen at partial pressures that fulfill the needs of oxidative phosphorylation (4). The authors concluded that substantial portions of the renal medulla may be operating on the verge of anoxia and therefore predisposed to anoxic damage. The present work, designed to test the possible relationship between the mTAL lesion and abnormal $\mathrm{O}_{2}$ delivery, confirms this prediction and shows that anoxia is the basis for mTAL injury in the isolated perfused kidney.

\section{Methods}

Male Sprague-Dawley rats, weighing $370-470 \mathrm{~g}$, fed on Purina rat chow (Ralston Purina Co., Chicago, IL) and allowed free access to water, were used for all experiments. Perfusion of the right kidney was performed according to the technique described by Ross et al. (5). Regular perfusion medium, as used in control rats, consisted of a Krebs-Ringer's-Henseleit solution with bovine serum albumin at a concentration of $6.7 \mathrm{~g} / 100$ $\mathrm{ml}$ and glucose at $5 \mathrm{mM}$, gassed with $5 \% \mathrm{CO}_{2}, 95 \% \mathrm{O}_{2}$.

1. Abbreviations used in this paper: GFR, glomerular filtration rate; mTAL, medullary thick ascending limb. 


\section{Experimental groups}

A. CONTROL GROUPS $(n=22)$. Kidneys were perfused with regular perfusion medium for either $15(n=8)$ or $90 \min (n=7)$. Control kidneys were fixed in situ to document normal mTAL morphology $(n=7)$.

B. HYPOXIC PERFUSIONS $(n=15)$. Kidneys were perfused with the same perfusion medium as control rats but gassed with $5 \% \mathrm{CO}_{2}$, $95 \% \mathrm{~N}_{2}$. The histology was observed after $15(n=8)$ or $90 \mathrm{~min}$ $(n=7)$ of perfusion.

15 min of perfusion are required in this model to allow hemodynamic parameters to stabilize and inulin to equilibrate. Therefore, the 15-min perfusion experiments were not used for the study of kidney function, but for the morphologic study of the earliest phase of the mTAL lesion and its time sequence under different conditions of regular and low oxygenation.

C. OXYGEN-ENRICHED PERFUSIONS $(n=22$ ). (These were 90min perfusions, gassed with $95 \% \mathrm{O}_{2}, 5 \% \mathrm{CO}_{2}$, unless specified otherwise.)

I. Erythrocytes $(n=10) .20-25 \mathrm{ml}$ of fresh blood from two heparinized donor rats was added to a regular perfusion medium within 1 min from the start of the perfusion at a final hematocrit of $13.6 \pm 0.4$. No filters were used for these experiments. A subset of two kidneys were perfused under the same conditions but gassed with $5 \% \mathrm{CO}, 5 \% \mathrm{CO}_{2}$, and $90 \% \mathrm{~N}_{2}$ to reduce $\mathrm{O}_{2}$ carrying capacity back to low levels.

II. Hemoglobin $(n=8)$. A purified, stable, unmodified hemoglobin solution $\left(\mathrm{P}_{50}=16\right.$ Torr) supplied by Dr. $\mathrm{R}$. Bolin (Department of the Army, Presidio of San Francisco, CA) was dialyzed against Krebs-Ringer's-Henseleit solution, concentrated by ultrafiltration, filtered, and added to the regular perfusion medium within $1 \mathrm{~min}$ from the start of the perfusion, at a final concentration of $3-5 \mathrm{~g} / 100 \mathrm{ml}$.

III. Oxypherol $(n=4)$. A fluorinated carbon (FC-43 emulsion, Oxypherol, Alpha-Therapeutic, Los Angeles, CA) was used as a blood substitute instead of the regular perfusion medium.

\section{Monitoring of oxygenation}

In all experimental groups, the $\mathrm{PO}_{2}$ of the perfusate was monitored using a blood gas monitor (PHM 73, Radiometer, Copenhagen). In groups A

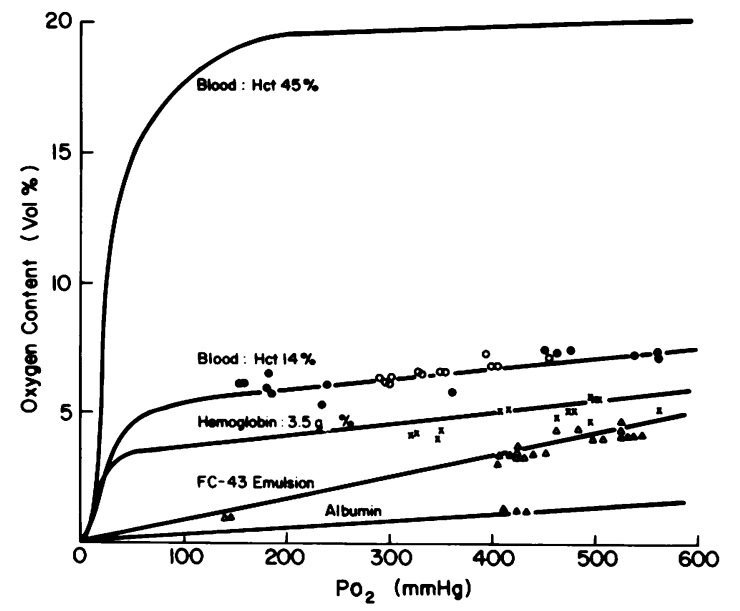

Figure 1. Expected oxygen dissociation curves for blood (hematocrit 45 and $14 \%$ ), hemoglobin, FC-43 emulsion, and albumin. The points represent actual measurements during perfusions with erythrocytes $(\bullet)$, hemoglobin 3-4 g/100 ml $(X)$ or 4-5 $\mathrm{g} / 100 \mathrm{ml}(O)$, FC-43 emulsion $(\Delta)$, and albumin $(\Delta)$. and $\mathrm{B}$, the $\mathrm{O}_{2}$ content was calculated using the constant of solubility of $\mathrm{O}_{2}$ in plasma, and confirmed by direct measurement in 10 randomly selected samples. In group $\mathrm{C}$ the $\mathrm{O}_{2}$ content was measured directly by volumetry using a Lex $\mathrm{O}_{2}$ con (Lexington Instrument Corp., Waltham, MA). As shown in Fig. 1 there was good concordance between the expected $\mathrm{O}_{2}$ contents and their actual measurements in the different groups.

\section{Morphologic techniques}

All kidneys of each control and experimental group were studied as separate groups for morphology. A three-way stopcock was incorporated into the circuit $5 \mathrm{~cm}$ from the arterial cannula to allow perfusion with the fixation solution, at the same pressure applied during the functional study, for an additional 5-8 min. The fixative solution contained $1.25 \%$ glutaraldehyde in $0.1 \mathrm{M}$ phosphate buffer ( $\mathrm{pH}$ 7.4). A 3-mm section of the outer medulla was removed, postfixed in $2 \% \mathrm{OsO}_{4}$, dehydrated and embedded in an araldite-epon 812 mixture. Large $1 \mu \mathrm{m}$ sections were cut to include the entire length of the mTAL. Selected blocks were examined by electron microscopy. In two additional kidneys perfused under regular conditions, special transverse sections (perpendicular to the axis of the vasa recta) were obtained to study the relationship of the mTAL lesion to vascular bundles.

The histological evaluation was completed by one of us in a "blinded" fashion; that is, without knowledge of experimental conditions. Three zones of the inner stripe were analyzed: upper third: all mTALs intersecting a line immediately adjacent to outer stripe (within $\sim 0.2 \mathrm{~mm}$ ); middle third: all mTALs intersecting a line drawn midway between the borders of the inner stripe; and lower third: all mTALs intersecting a line immediately adjacent to the inner medulla (within $\sim 0.2 \mathrm{~mm}$ ).

These points were chosen for analysis because they provided areas in which topographical landmarks were easily ascertained. A percentage score was used to indicate the fraction of tubules involved with minimal to mild (chromatin margination, minor degrees of mitochondrial swelling), moderate (blatant mitochondrial swelling with limited nuclear pyknosis), or severe (blatant mitochondrial swelling with extensive nuclear pyknosis and cell fragmentation) changes. Between 68 and 272 tubules (mean 137) were evaluated per kidney in a total of 61 kidneys.

The data are presented as mean \pm SEM. Statistical analysis was by the $t$ test, unless specified otherwise.

\section{Results}

\section{Renal functions in the different groups}

The functional parameters are summarized in Table I. In group B (hypoxic perfusion) a marked decrease in oxygen content was associated with low GFR and reduced fractional sodium reabsorption. The oxygen content of the perfusate was substantially increased in all $C$ subgroups and most pronounced with erythrocytes $\left(C_{1}\right)$. This group demonstrated improved conservation of sodium and potassium, as compared with perfusions containing albumin alone (group A).

The rate of flow of perfusate to the kidney was significantly lower (and calculated renal resistance correspondingly higher) in the experiments of group $\mathrm{C}$ than in the control group (group A).

\section{Morphological observations}

Fig. 2 illustrates the injury to the mTAL as consistently seen in isolated kidneys perfused with regular medium (Fig. $2 \mathrm{~B}$ - 
Table I. Oxygenation and Function of the Kidneys in the Different Groups

\begin{tabular}{|c|c|c|c|c|c|c|}
\hline & $\begin{array}{l}\text { Arterial } \\
\mathrm{PO}_{2}\end{array}$ & $\begin{array}{l}\text { Arterial } \mathrm{O}_{2} \\
\text { content }\end{array}$ & GFR & $\mathrm{TR}_{\mathrm{N}_{\bullet}}$ & $\mathrm{FE}_{\mathbf{K}}$ & $\begin{array}{l}\text { Renal } \\
\text { perfusion flow }\end{array}$ \\
\hline & $m m H g$ & $\mathrm{vol} / 100 \mathrm{ml}$ & $\mathrm{ml} / \mathrm{min}$ & $\%$ & $\%$ & $\mathrm{ml} / \mathrm{min}$ \\
\hline A (controls) $(n=7)$ & $495 \pm 12$ & $1.5 \pm 0.04$ & $0.52 \pm 0.06$ & $93.9 \pm 1.3$ & $58.2 \pm 11.5$ & $45.7 \pm 2.6$ \\
\hline \multicolumn{7}{|l|}{ B (low oxygen perfusions) } \\
\hline$(n=7)$ & $38 \pm 1^{*}$ & $0.12 \pm 0.004^{*}$ & $0.12 \pm 0.02 \ddagger$ & $45.7 \pm 1.2^{*}$ & $76.9 \pm 4.1$ & $37.6 \pm 1.9$ \\
\hline$C_{1}$ (erythrocytes) $(n=8)$ & $463 \pm 18$ & $7.1 \pm 0.3^{*}$ & $0.54 \pm 0.1$ & $97.4 \pm 0.5 \S$ & $26.9 \pm 2.9 \S$ & $10.5 \pm 2.0^{*}$ \\
\hline \multicolumn{7}{|c|}{$C_{l}$ (erythrocytes with $5 \% \mathrm{CO}$ ) } \\
\hline$(n=2)$ & 36.5 & 0.11 & 0.17 & 44.5 & 72.3 & 10.0 \\
\hline $\mathrm{C}_{\mathrm{II}}$ (hemoglobin) $(n=8)$ & $460 \pm 10$ & $5.8 \pm 0.3^{*}$ & $0.76 \pm 0.2$ & $96.5 \pm 0.4$ & $22.6 \pm 3.6 \S$ & $24.4 \pm 1.4^{*}$ \\
\hline $\mathrm{C}_{\mathrm{III}}$ (Oxypherol) $(n=4)$ & $513 \pm 8$ & $4.3 \pm 0.1^{*}$ & $0.25 \pm 0.1$ & $97.9 \pm 0.8$ & $10.6 \pm 4.5 \S$ & $13.4 \pm 1.4^{*}$ \\
\hline
\end{tabular}

Data are given at $60 \mathrm{~min}$. $\mathrm{TR}_{\mathrm{NA}}$, tubular reabsorption of sodium; $\mathrm{FE}_{\mathrm{K}}$, fractional excretion of potassium.

$* P<0.001$, vs. group A.

$\ddagger P<0.005$, vs. group $\mathrm{A}$.

$\S P<0.02$, vs. group $\mathrm{A}$.

in area $B$ and $C$ were affected by the injury, and showed moderate to severe damage both at 15 and 90 min (Figs. 3 and 5). Thus, hypoxic perfusion both exacerbated the damage and markedly

$E$ ), as compared with kidneys fixed in situ (Fig. $2 A, 2 E$ inset). As damage proceeds, nuclei become more obvious and chromatin margination occurs. Later, mitochondrial swelling appears, and finally the nucleus condenses (pyknosis) and the luminal membrane becomes fragmented. These changes correspond to the well-described sequence that is characteristic of ischemic cell injury (6). They can all be perceived by light microscopy in $1-\mu \mathrm{m}$ sections, and are easily apparent in electron microphotographs. When limited detail was perceived in the cell in such sections (nuclei and mitochondria not easily visible, and the cell border was intact; e.g., Figs. 4, 7A), electron microscopy invariably revealed minimal or no damage.

\section{Group A: gradients of $m T A L$ damage}

Evaluation of the severity of the lesion in the control group at both 15 and 90 min showed that it was distributed along two types of anatomical gradients, corresponding to the expected gradients of oxygen tension in the renal medulla.

Axial gradient. The most severe and consistent damage was located in the deepest portions of the mTAL, close to the inner medulla. The least severe damage, frequently absent, was found in the most superficial third of the mTAL, near its transition to the cortical thick ascending limb (Fig. 3). The damage increased mostly in its severity from 15 to $90 \mathrm{~min}$ (Fig. 3).

Transverse gradient and polarity. The lesion was consistently more advanced in the mTALs located most distant from the vasa recta bundles. Indeed, epithelium immediately adjacent to the vascular bundle was frequently preserved, while opposed cells in the same tubules were markedly affected (Fig. 4). These results suggest that proximity to oxygen supply influences the development of the lesion.

Group B: effects of low oxygen perfusion

Low oxygen perfusion greatly enhanced the extent and severity of the mTAL lesion. Most mTALs in area A and all mTALs attenuated its gradations (Fig. 3).

\section{Group C: effects of $\mathrm{O}_{2}$ enriched perfusion}

In all perfusions supplemented with erythrocytes the lesion was conspicuously absent (Fig. 6). In two kidneys gassed with carbon monoxide, $\mathrm{O}_{2}$ content was $<1 \mathrm{vol} / 100 \mathrm{ml}$ and the lesion reappeared, despite the presence of erythrocytes (Fig. 6). During perfusion with hemoglobin the mTAL lesion was again prevented (Figs. 7 and 8). With Oxypherol the injury was remarkably attenuated but still present (Figs. 7 and 8 ).

The relationship between the extent and severity of the mTAL lesion and the $\mathrm{O}_{2}$ content of the perfusion is summarized in Fig. 8. The typical lesion is found during regular perfusion at an $\mathrm{O}_{2}$ content $\sim 1.5 \mathrm{vol} / 100 \mathrm{ml}$. A 10 -fold decrease in the $\mathrm{O}_{2}$ content (in the low $\mathrm{O}_{2}$ perfusions) transforms the lesion to extensive and almost complete destruction of the mTAL. Conversely, increasing the $\mathrm{O}_{2}$ content to $4-5 \mathrm{vol} / 100 \mathrm{ml}$ with $\mathrm{Ox}$ ypherol affords significant protection, while the lesion is no longer detected above an $\mathrm{O}_{2}$ content of 6-7 vol/100 ml, with hemoglobin or erythrocytes.

\section{Discussion}

Leichtweiss et al. (7) have previously shown that the tissue partial pressure of $\mathrm{O}_{2}$, as measured by platinum microelectrodes inserted into the rat kidney from cortex to medulla, drops steeply in the cortico-medullary junction to values clustered around $10 \mathrm{mmHg}$ in the medulla, both in vivo and during isolated perfusion (calculating from the depth of electrode penetration in Leichtweiss's studies, this $\mathrm{PO}_{2}$ was probably recorded at the level of the inner stripe of the outer medulla). It has been proposed (8) that the inner medulla functions adequately in this hypoxic environment because of its reliance on both aerobic and anaerobic glycolysis. However, recently, the concept of cellular hypoxia of the renal outer medulla has been suggested, using direct measurement 

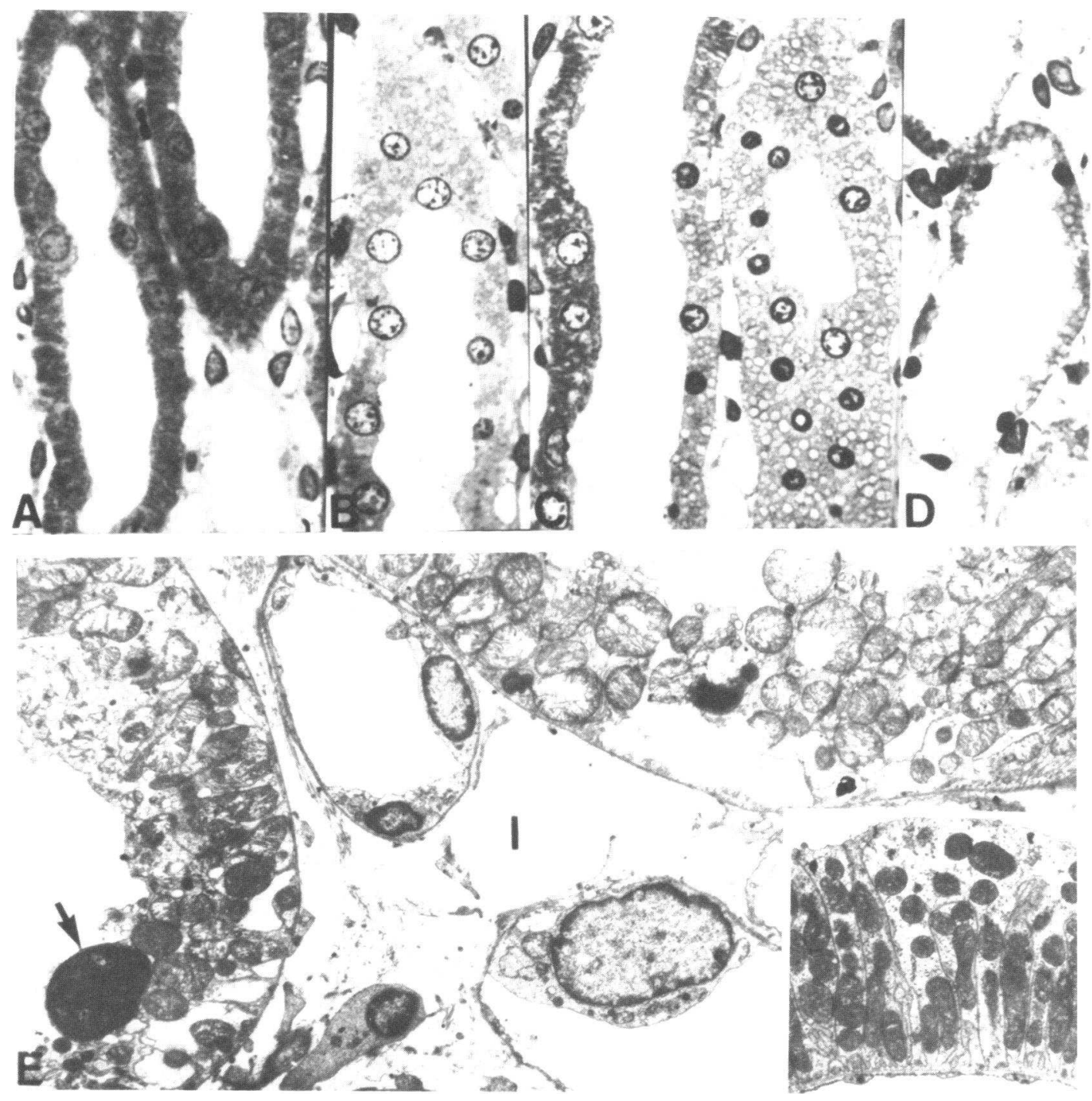

Figure 2. A consistent sequence of cellular injury as seen during isolated kidney perfusion can be readily recognized in $1-\mu \mathrm{m}$ plastic sections. In control material $(A)$ (kidneys perfused with fixative in situ), mTAL mitochondria can be defined as rod-shaped elements and the normal nuclear chromatin distribution can be appreciated. In early phases of injury, chromatin margination occurs that later is associated with mitochondrial swelling. In $B$, earlier phases of mitochondrial swelling can be perceived especially in the tangential section of the mTAL (upper). Mitochondrial swelling is more evident in $C$ (right) where chromatin margination is also marked. mTAL epithelium in $C$ (extreme left) has a mild degree of chromatin

by optical spectroscopy of the redox state of cytochrome $a, a_{3}$, the terminal electron carrier of the mitochondrial chain, which transports electrons directly to oxygen. The oxidative state of this enzyme in rat kidneys was estimated as at least $20 \%$ reduced in vivo (9) and $30-40 \%$ reduced during isolated perfusion (3), indicating that despite high $\mathrm{PO}_{2}$ in artery and vein, substantial margination associated with minimal mitochondrial swelling. The epithelium of the TAL in $D$ shows the final stages of cell injury. The cells are fragmented and luminal membranes cannot be defined; nuclear pyknosis and mitochondrial swelling are present. Electron microscopy of such tubules as noted in $(E)$ reveal nuclear pyknosis (arrow), marked mitochondrial swelling, and loss of luminal and basal membranes. In sharp contrast, elements contained within the interstitium $(I)$ have relatively intact nuclear and membrane components. The inset shows, by comparison, an electron micrograph of a normal kidney fixed in situ. $\times 640(A-D), \times 3,000$ ( $E$ and inset).

portions of the kidney may exist in an hypoxic milieu. Since inhibitors of cortical metabolism did not affect the redox state of the cytochrome, whereas furosemide or bumetanide produced a significant further oxidation of cytochrome $a, a_{3}$, it appeared that the mTAL was an important site of reduced cytochrome oxidase, so that cells of the mTAL might operate on the brink 


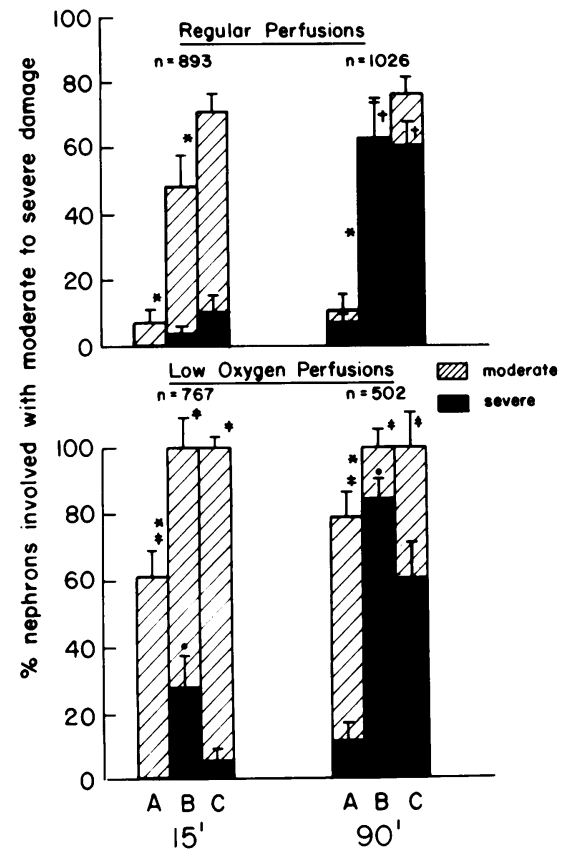

Figure 3. (Upper panel) Axial gradient of mTAL damage at 15 and $90 \mathrm{~min}$ of regular perfusion. The extent of mTAL damage in the inner stripe of the outer medulla was evaluated in three regions: outer $(A)$, mid $(B)$, and inner $(C)$ (see Methods). Increasing severity of damage is noted progressing from the most superficial aspect of the inner stripe to the zone located adjacent to the inner medulla. Increasing severity of damage is also noted from 15 (mostly moderate) to $90 \mathrm{~min}$ (mostly severe). (Lower panel) Attenuated axial gradient of mTAL damage at 15 and 90 min of low oxygen perfusion. Striking extension of the lesion to all nephrons in areas $B$ and $C$ and most nephrons of area $A$ is noted. Increasing severity of damage is mainly seen in area $B$ at 15 and $90 \mathrm{~min}$, as compared with regular perfusions. $n$ represents total number of tubules evaluated in each group; ${ }^{*}$, refers to comparison of adjacent columns; $P<0.05$; $\dagger$, refers to comparison of respective columns at 90 vs. $15 \mathrm{~min}, P$ $<0.001$; $\ddagger$ and $\bullet$, refer to comparison of respective columns of low oxygen perfusions vs regular perfusions, $P<0.001$ and $<0.05$, respectively. $P$ was calculated by Mann-Whitney $U$ test.

of anoxia during isolated perfusion or eventually in vivo during ischemia.

The present study demonstrates that the selective and reproducible damage to the $\mathrm{mTAL}$ in isolated rat kidney perfusion (1) is a lesion of anoxia. The observation that the damage is most severe in areas removed from vascular bundles and near the inner medulla, areas likely to suffer from poor $\mathrm{O}_{2}$ supply, itself suggests $\mathrm{O}_{2}$ deficiency as a key factor. (Since the mTAL of the short-looped nephrons are most removed from the vascular bundle (10), these nephrons are singularly vulnerable to limited availability of $\mathrm{O}_{2}$.) Indeed, the demonstration of these gradients of damage provides a remarkable morphological counterpart to the axial (cortico-medullary) gradient of tissue $\mathrm{PO}_{2}$ shown by
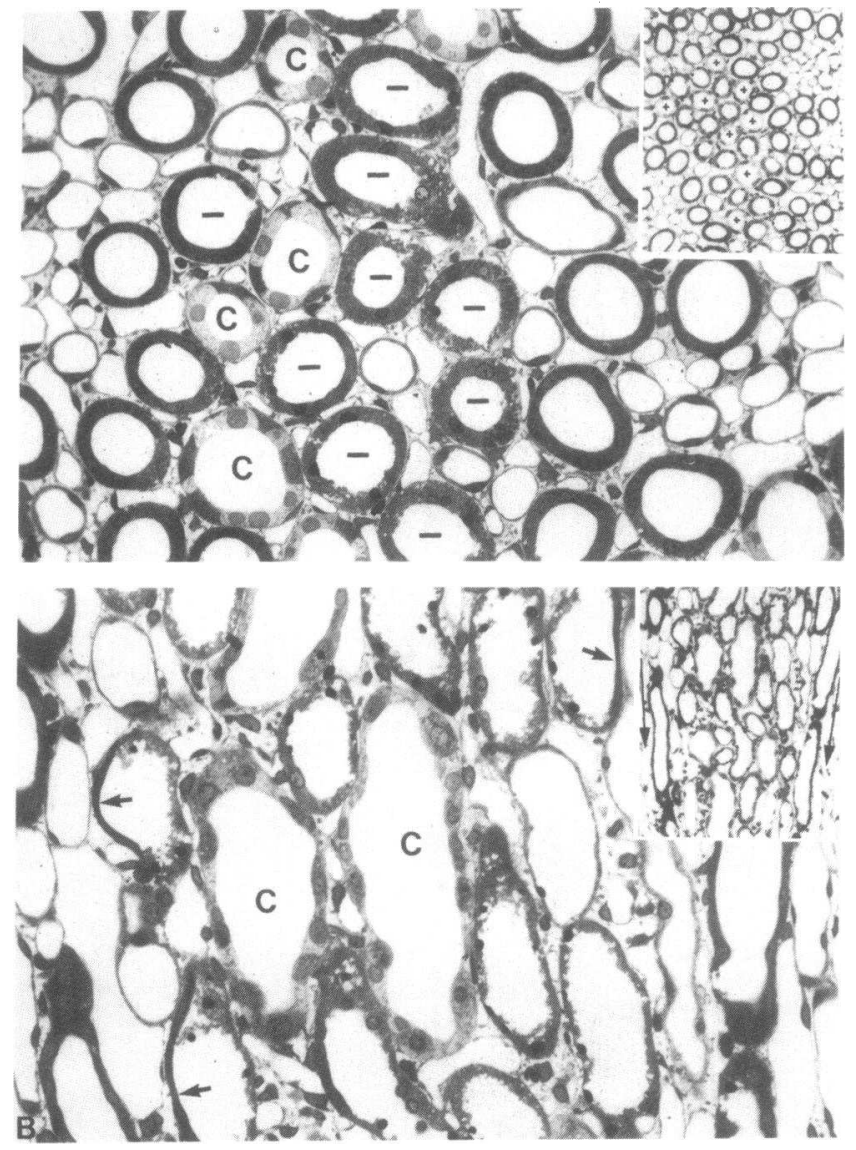

Figure 4. Transverse gradient of mTAL damage (as seen at $90 \mathrm{~min}$ ). In $A$, low power (inset) reveals the tubular-vascular organization of the inner stripe in horizontal section. The collecting ducts $(+)$ are centrally located between vascular bundles which are located in the upper right and lower left. The higher power $(A)$, with vascular bundles at lateral aspects shows that the greatest mTAL changes (bars) generally occur near collecting ducts $(C)$ and in zones most distant from vascular bundles. In $B$, the low power sagittal section (inset) also shows maintenance of mTAL epithelia adjacent to the vascular bundles (arrows). Higher power $(B)$ of zone between arrows reveals that the polarity of the mTAL damage is such that epithelium (arrows) adjacent to the vascular bundle is preserved while opposed cells within the same tubule are severely injured. The most severe mTAL damage again is related to collecting ducts $(C) . A$ and $B$, $\times 250$; insets, $\times 65$.

Leichtweiss et al. (7) and to the transverse (vasculo-cellular) gradient of $\mathrm{PO}_{2}$ suggested by Epstein et al. (3).

The effacement of these gradients, and their replacement by a lesion involving all mTALs in their entire length as early as $15 \mathrm{~min}$ of hypoxic perfusion support the concept that the regular mTAL lesion seen consistently during perfusion with albumin is a lesion of anoxia, preferentially affecting cells that have high oxygen demand and are removed from oxygen supply. The high selectivity of this injury is emphasized by the findings 


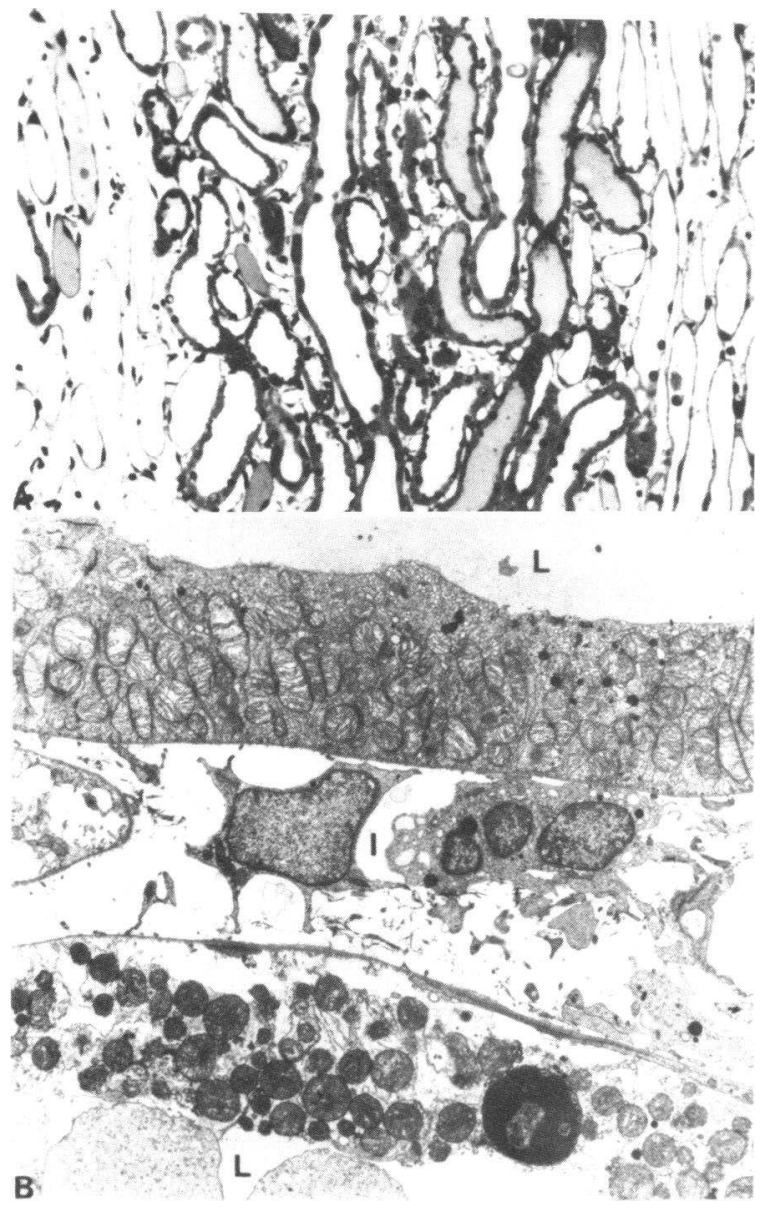

Figure 5. If the perfusate has a very limited $\mathrm{O}_{2}$ content $(A)$, all mTAL have moderate to severe injury. Fragmentation and varying degrees of nuclear pyknosis are common; the polarity of injury is essentially abolished. Vascular bundles are at lateral aspects. $(B)$ In this electron micrograph, two mTAL are present with lumens $(L)$ at the upper and lower aspect. The interstitium area $(I)$ contains two relative unremarkable interstitial cells. Both mTAL show marked mitochondrial swelling, but in the lower tubule luminal membrane fragmentation and nuclear pyknosis are noted as well. These changes seen in hypoxic perfusion beginning at $15 \mathrm{~min}$ are identical to the type of injury observed after 90 min of perfusion with normal oxygenation. $\times 140 ; \times 2,800$.

at $90 \mathrm{~min}$ of hypoxic perfusion, where extensive and diffuse tubular destruction was still basically confined to the mTAL.

Measures to increase medullary $\mathrm{O}_{2}$ delivery resulted in a marked improvement related to $\mathrm{O}_{2}$ content of the perfusate. Supplementation of the albumin perfusate with fresh rat blood to a hematocrit of $14 \%$ resulted in complete preservation of the mTAL integrity. To minimize hemolysis, erythrocytes were not separated but supplementation with plasma per se did not provide any diminution of the mTAL lesion (data not shown). Moreover, carbon monoxide, which markedly reduced $\mathrm{O}_{2}$ bind-

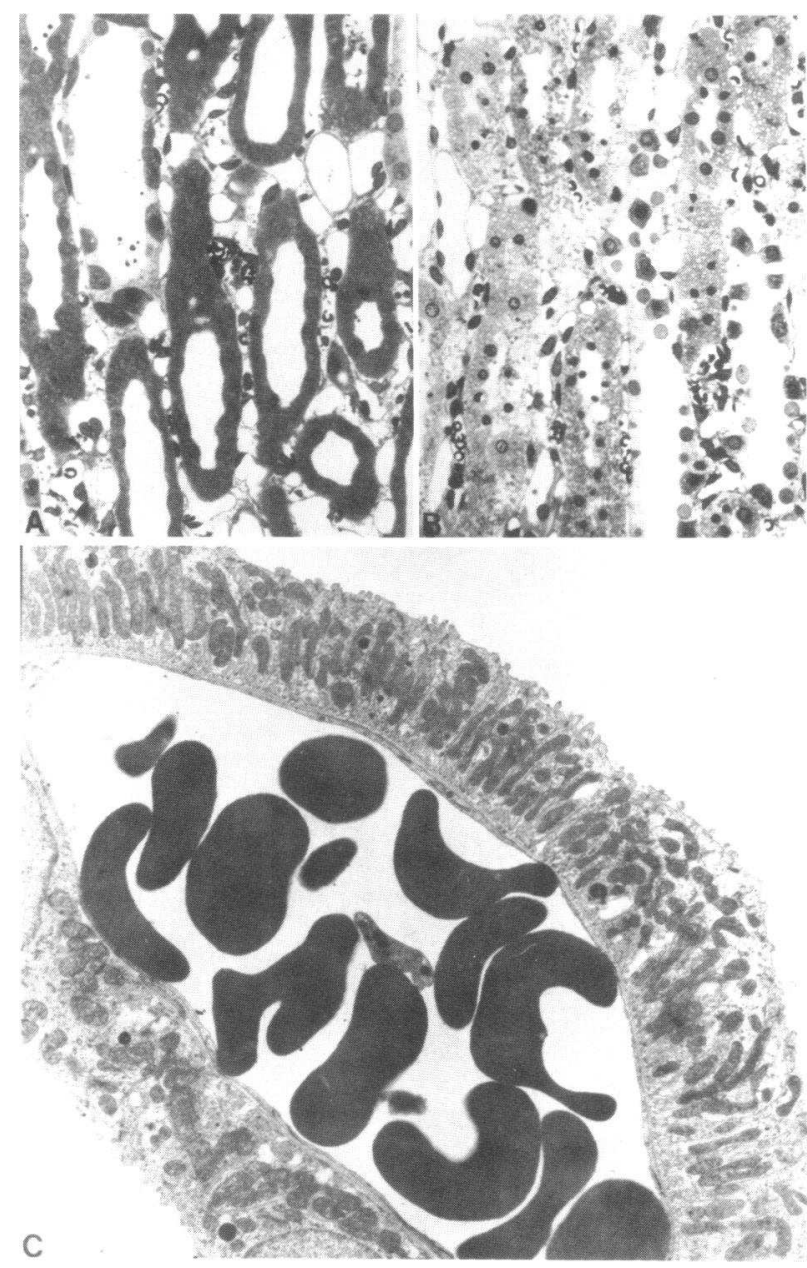

Figure 6. If the perfusate contains erythrocytes, the mTAL is well preserved $(A)$. However, if the $\mathrm{O}_{2}$-carrying capacity is reduced by gassing with $\mathrm{CO}, \mathrm{mTAL}$ damage is again observed $(B)$. Electron microscopy confirms the integrity of mTAL when oxygenated erythrocytes are included in the perfusate $(C)$. The normal appearance of these mitochondria contrasts with the mTAL in kidneys perfused without erythrocytes (Fig. $2 E$ ). Two wellmaintained $\mathrm{mTAL}$ are noted (right, lower left) with a centrally located interstitial capillary filled with erythrocytes. $\times 200, \times 200$, $\times 1,800$.

ing to hemoglobin, reversed the protection afforded during erythrocyte perfusions, underlining the dependence of this protection on improved oxygen-carrying capacity. Additional verification of this hypothesis was afforded by the hemoglobin experiments. Nearly complete protection was provided by supplementing the albumin perfusate with a purified hemoglobin solution possessing improved oxygen-carrying capacity. Finally, increased oxygen dissolution in the fluorinated carbon blood substitute FC 43 emulsion also led to significant protection of the mTAL, again suggesting improved oxygen delivery to the mTAL as the key factor in its protection. 


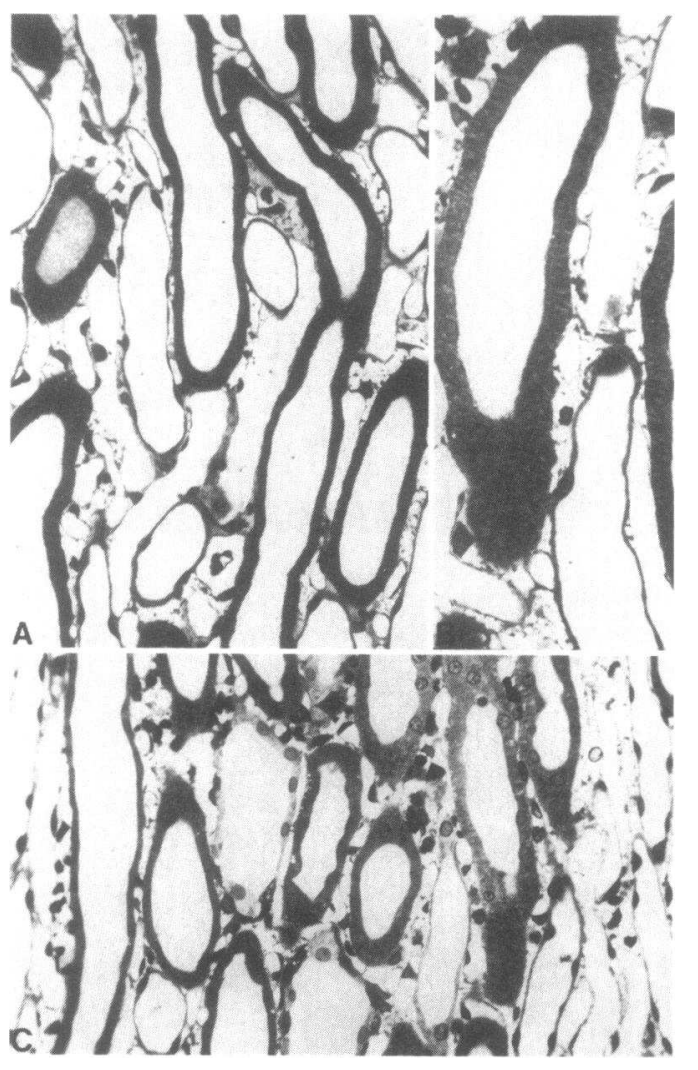

Figure 7. If hemoglobin is included in the perfusate, the mTAL is preserved $(A$ and $B)$. Oxypherol $(C)$ also attenuates the injury, but mild to moderate damage can still be recognized in some mTALs $($ right $) . \times 400, \times 640 ; \times 400$.

Increased conservation of potassium seen in the three types of $\mathrm{O}_{2}$-enriched perfusions (Table $\mathrm{I}$ ) may be related to a diminution of solute delivery to the distal tubule during isolated perfusion (11), owing to improved reabsorption of $\mathrm{NaCl}$ in the mTAL. Fractional reabsorption of sodium did in fact tend to be higher in the high oxygen perfusions of group $\mathrm{C}$, significantly so in Group $C_{1}$. This tendency may well have been opposed by the obligatory urinary excretion of hemoglobin dimers in group $\mathrm{C}_{\mathrm{II}}$ and of perfluorotributylamine ${ }^{2}$ in group $\mathrm{C}_{\mathrm{III}}$. Similar improvements in sodium conservation have already been reported with erythrocytes or fluorinated carbon perfusions $(12,13)$. Normalization of the solute reabsorption is not expected when glucose is used as the only substrate (2). In several experiments where amino acids (2) were included in addition to erythrocytes or hemoglobin, a fractional reabsorption of $99 \%$ or higher was observed. Preliminary observations suggest that preserved mTAL morphology is also associated with improved concentrating ability (unpublished observations).

2. Perfluorotributylamine has a mol wt of 660 , is present in FC 43 at a concentration of $300 \mathrm{mM}$, and may dissociate from emulsion particles to produce an osmotic diuresis.

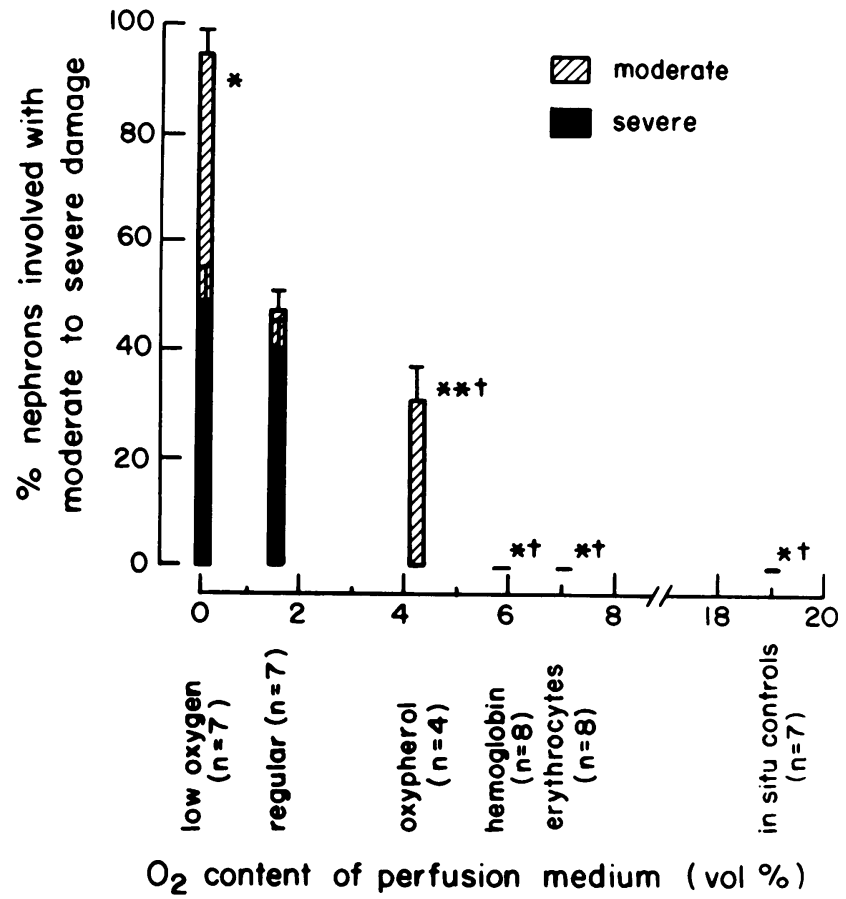

Figure 8. Relationship between degree of mTAL damage and oxygenation of perfusion medium. The quantitation of damage is for the whole inner stripe of the outer medulla (areas $A, B$, and $C$ combined). ${ }^{*}$, refers to extent (moderate or severe); $\dagger$, to severity of damage compared with regular perfusions; both to $P<0.001$. **, $P$ $<0.05$.

The steep cortico-medullary gradient of $\mathrm{PO}_{2}$ in kidney tissue is the result of the medullary vascular structure and the conditions imposed by the counter-current exchange system (14). The hairpin conformation of the vasa-recta prevents the dissipation of a cortico-medullary gradient of osmolality, but at the cost of counter-current diffusion of $\mathrm{O}_{2}$ between arterial and venous limbs. The high $\mathrm{O}_{2}$ consumption of this zone keeps medullary $\mathrm{PO}_{2}$ low and almost undisturbed by axial flow. During isolated perfusion, the high arterial $\mathrm{PO}_{2}$ favors arteriovenous diffusion, leaving medullary $\mathrm{PO}_{2}$ in the same low range as found in vivo (7) $(\sim 10 \mathrm{mmHg})$. Blood or perfusate flowing through the vasa recta of the outer medulla, therefore, will have its $\mathrm{PO}_{2}$ rapidly lowered to this range. At this $\mathrm{O}_{2}$ tension, because of the sigmoid shape of the $\mathrm{O}_{2}$ dissociation curve, blood still has a significant $\mathrm{O}_{2}$ content, whereas the amount of $\mathrm{O}_{2}$ dissolved in an albumin solution is extremely low, as illustrated in Table II. As a result, oxygen delivery to the medulla is inevitably compromised during isolated perfusion without hemoglobin. The elevated rate of perfusion and high content of dissolved $\mathrm{O}_{2}$ in the perfusate may serve to maintain delivery of oxygen to cortical tissues close to that in vivo, but such compensation cannot be achieved in the medulla, which consequently suffers anoxic damage.

It is conceivable that the marked increase in renal perfusion flow seen in the isolated kidney perfused with albumin is, at 
Table II. Estimated Oxygen Delivery to Whole Rat Kidney and to Outer Medulla In Vivo and during Isolated Perfusion

\begin{tabular}{|c|c|c|c|c|c|}
\hline & $\begin{array}{l}\text { Arterial } \\
\mathrm{PO}_{2}\end{array}$ & $\begin{array}{l}\text { Arterial } \mathrm{O}_{2} \\
\text { content }\end{array}$ & Flow & $\mathrm{O}_{2}$ delivery & mTAL lesion \\
\hline & $\mathrm{mmHg}$ & vol\% & $\mathrm{ml} / \mathrm{min} / \mathrm{g}$ & $\mathrm{ml} / \mathrm{min} / \mathrm{g}$ & \\
\hline \multicolumn{6}{|l|}{ Whole kidney } \\
\hline $\begin{array}{l}\text { Kidney in vivo } \\
\text { Isolated perfused kidney" }\end{array}$ & $70-90^{*}$ & $18-19^{*}$ & $3-5 \ddagger$ & $0.5-0.9$ & Never seen§ \\
\hline (albumin) & $500-600$ & $1.2-1.8$ & $24-28$ & $0.3-0.5$ & Always seen \\
\hline \multicolumn{6}{|l|}{ Outer medulla } \\
\hline $\begin{array}{l}\text { Kidney in vivo } \\
\text { Isolated perfused kidney }\end{array}$ & $25 \pi$ & $4^{* *}$ & $2 \ddagger$ & 0.08 & Never seen \\
\hline (albumin) & $25 \pi$ & 0.06 & $13 \neq \ddagger$ & 0.008 & Always seen \\
\hline
\end{tabular}

\footnotetext{
* As determined in several control rats under anesthesia.

$\ddagger$ See reference 10 .

$\S$ Normal mTAL morphology was observed in seven control kidneys fixed in situ by glutaraldehyde perfusion.

"From references 1 and 2 , and present work.

T The upper range of tissue $\mathrm{PO}_{2}$ in the outer medulla as measured by Leichtweiss et al. (7) was chosen as an estimate of arterial $\mathrm{PO}_{2}$ in this region.

** Estimate from reference 15 .

$\ddagger$ Cortical and medullary flow are thought to be increased in roughly equal proportions during isolated perfusion $(16,17)$.
}

least in part, a physiological response to the low $\mathrm{O}_{2}$ supply. This vasodilation was indeed remarkably blunted in all $\mathrm{O}_{2}$-enriched perfusions (Table I) where normal renal innervation and renin substrate supply were absent. The effects of erythrocytes on perfusate viscosity are minimal below a hematocrit of 30 as measured either in vivo or in vitro (18) and are unlikely to be the explanation of these striking changes in flow. In other experimental models, acute anemia increases renal blood flow (19), whereas hyperbaric oxygenation decreases renal blood flow (20), supporting the concept that $\mathrm{O}_{2}$ availability might be a factor in the regulation of renal blood flow, as shown in other tissues (21). Prostaglandins could theoretically mediate this hypoxic renal vasodilation, but indomethacin does not prevent its occurrence during the first hour of perfusion (G. Kershaw and J. Stoff, unpublished observations), and their role remains to be proven. This singular sensitivity of the medullary portion of the kidney to $\mathrm{O}_{2}$ content, at constant arterial $\mathrm{PO}_{2}$, might also make it an ideal site for the regulation of erythropoietin synthesis.

We have recently shown that decreasing the work of transport and thereby reducing the demand for oxygen by mTAL cells can prevent the mTAL lesion. Perfusion with furosemide or ouabain or with hyperoncotic albumin medium (to halt glomerular filtration) consistently reduce or abolish the anoxic damage to the mTAL (22). (Similarly, the addition of amino acids to the perfusate attenuates the mTAL lesion [2], probably by improving proximal tubular reabsorption ["unloading" the mTAL] and by increasing renal perfusion flow [reference 2 , and unpublished observations] with the net result of a combined decrease in $\mathrm{O}_{2}$ consumption and increase in $\mathrm{O}_{2}$ delivery in the
mTAL.) These experiments appear to be examples in the kidney of the general principle, so familiar in the pathophysiology of the heart, that cellular work accelerates anoxic injury and that an imbalance between oxygen supply and demand is a prelude to cell damage. It is not surprising that the cells of the mTAL, by virtue of their high rate of metabolism (23) and their special location with limited $\mathrm{O}_{2}$ supply, are so exquisitely susceptible to anoxia. They might therefore be expected to be especially vulnerable in vivo to ischemia as well.

Several reports have, in fact, indicated a defect in TAL function in experimental models of ischemic renal failure (2426). Structural changes including mitochondrial swelling and nuclear pyknosis have been identified in the mTALs of kidneys examined from rats submitted to hemorrhagic hypotension (27) or to glycerol injections (28). Decreased concentrating ability (one of the most consistent defects in human acute tubular necrosis), polyuria (in nonoliguric renal failure), and activation of tubuloglomerular feedback (29) could all be direct consequences of a selective mTAL ischemia. Focal or diffuse TAL necrosis in acute renal failure has been reported by several observers $(30-34)$. The protection afforded by the prophylactic administration of furosemide in several models of ischemic acute tubular necrosis has never been clearly explained (35) but evokes an intriguing parallel to the prevention of MTAL anoxic damage during isolated perfusion by decreasing $\mathrm{O}_{2}$ demand by cells. Indeed, the redistribution of cortical flow in favor of the medulla, an early event in hypotension (36), and the profound disproportionate fall in GFR in acute renal failure might be viewed, at least in part, as a regulatory mechanism developed to protect 
the mTAL from the dangerous and precarious equilibrium between $\mathrm{O}_{2}$ demand and supply within the outer medulla of the kidney.

The selective vulnerability of mTAL cells to anoxic damage, so clearly demonstrable in isolated perfused kidneys, might influence their fragility in other experimental models (isolated TAL tubules, isolated TAL cells) where careful oxygenation may be crucial. Finally, the specific susceptibility of the mTAL to anoxia may play a key role in a variety of disease states characterized by renal medullary ischemia (37).

\section{References}

1. Alcorn, D., K. R. Emslie, B. D. Ross, G. B. Ryan, and J. D. Tange. 1981. Selective distal nephron damage during isolated kidney perfusion. Kidney Int. 19:638-647.

2. Epstein, F. H., J. T. Brosnan, J. D. Tange, and B. D. Ross. 1982. Improved function with amino acids in isolated perfused kidney. Am. J. Physiol. 243:F284-292.

3. Epstein, F. H., R. S. Balaban, and B. D. Ross. 1982. Redox state of cytochrome $a, a_{3}$ in isolated perfused rat kidney. Am. J. Physiol. 243:F356-363.

4. Balaban, R. S. 1982. Non-invasive techniques for the investigation of renal metabolism and transport. Biochemistry of Kidney Functions. INSERM Symposium No. 21. F. Morel, editor. Elsevier Biomedical Press, Amsterdam. 337-349.

5. Ross, B. D., F. H. Epstein, and A. Leaf. 1973. Sodium reabsorption in the perfused rat kidney. Am. J. Physiol. 225:1165-1171.

6. Trump, B. F., I. K. Berezesky, and R. A. Cowley. 1982. The cellular and subcellular characteristics of acute and chronic injury with emphasis on the role of calcium. In Pathophysiology of Shock, Anoxia and Ischemia. R. A. Cowley and B. F. Trump, editors. The Williams \& Wilkins Co., Baltimore. 6-46.

7. Leichtweiss, H. P., D. W. Lubbers, C. H. Weiss, H. Baumgarth, and W. Reschke. 1969. The oxygen supply of the rat kidney measurement of intrarenal $\mathrm{pO}_{2}$. Pfluegers Arch. 309:328-349.

8. Cohen, J. J. 1979. Is the function of the renal papilla coupled exclusively to an anaerobic pattern of metabolism? Am. J. Physiol. 236:F423-433.

9. Balaban, R. S., and A. L. Silvia. 1981. Spectrophotometric monitoring of $\mathrm{O}_{2}$ delivery to the exposed rat kidney. Am. J. Physiol. 241:F257262.

10. Jamison, R. L., and W. Kriz. 1982. Structure of the medulla as a whole. In Urinary Concentrating Mechanisms. Oxford University Press, New York. 55-76, 259-271.

11. De Mello, G., and T. Maack. 1976. Nephron function of the isolated perfused rat kidney. Am. J. Physiol. 231:1699-1707.

12. Swanson, J. W., A. Besarab, P. P. Pomerantz, and A. DeGuzman. 1981. Effect of erythrocytes and globulin on renal functions of the isolated rat kidney. Am. J. Physiol. 241:F139-150.

13. Franke, H., M. MaPyusz, and D. Runge. 1978. Improved sodium and PAH transport in the isolated fluorocarbon-perfused rat kidney. Nephron. 22:423-431.

14. Cohen, J. J., and D. E. Kamm. 1981. Renal metabolism: relation to renal function. In The Kidney. B. Brenner, and F. C. Rector, editors. W. B. Saunders Co., Philadelphia. 155-160.

15. Altman, P. L., and D. S. Dittmer. 1961. Blood and other body fluids. Federation American Society Experimental Biology, Washington, DC. 153.
16. Ross, B. D. 1978. The isolated perfused rat kidney. Clin. Sci. Mol. Med. 55:513-521.

17. Rubin, J. E., J. S. Stoff, P. Silva, and F. H. Epstein. 1976. Distribution of regional flow to cortex of the isolated perfused rat kidney: effects of norepinephrine. Kidney Int. 10:597.

18. Whittaker, S. R. F., and F. R. Winton. 1933. The apparent viscosity of blood flowing in the isolated hind limb of the dog, and its variation with corpuscular concentration. J. Physiol. (Lond.). 78:338369.

19. Grupp, I., G. Grupp, J. C. Holmes, and N. O. Fowler. 1972. Regional blood flow in anemia. J. Appl. Physiol. 33(4):456-461.

20. Norman, J. N., J. R. Shearer, A. J. Napper, I. Robertson, and G. Smith. 1974. Action of oxygen on the renal circulation. Am. J. Physiol. 227:740-799.

21. Sparks, H. V. Jr., and F. L. Belloni. 1978. The peripheral circulation: local regulation. Ann. Rev. Physiol. 40:67-92.

22. Brezis, M., S. Rosen, P. Silva, and F. H. Epstein. 1984. Transport activity modifies thick ascending limb damage in the isolated perfused kidney. Kidney Int. In press.

23. Eveloff, J., E. Bayerdorffer, P. Silva, and R. Kinne. 1981. Sodiumchloride transport in the thick ascending limb of Henle's loop. Pfluegers Arch. 389:263-270.

24. Mason, J., H. U. Gutsche, L. Mooze, R. Muller-Suur, S. Patz, and I. Lichtenstein. 1979. The early phase of experimental acute renal failure IV. The diluting ability of the short loops of Henle. Pfluegers Arch. 379:11-18.

25. Hanley, M. J. 1980. Isolated nephron segments in a rabbit model of ischemic acute renal failure. Am. J. Physiol. 239:F17.

26. Anderson, R. J., J. A. Gordon, J. Kim, L. M. Peterson, P. A. Gross, V. VanPutten, and M. Ellis. 1982. Renal concentration defect following non-oliguric acute renal failure in the rat. Kidney Int. 21:583591.

27. Kreisberg, J., R. E. Bulger, B. F. Trump, and R. B. Nagle. 1976. Effects of transient hypotension on the structure and function of rat kidney. Virchows Arch. B Cell Pathol. 22:121-133.

28. Suzuki, T., and F. K. Mostofi. 1970. Electron microscopic studies of acute tubular necrosis. Lab. Invest. 23:15-28.

29. Thurau, K., and J. W. Boylan. 1976. Acute renal success. The unexpected logic of oliguria in acute renal failure. Am. J. Med. 61:308315.

30. Bywaters, E. G. L., and J. H. Dible. 1942. The renal lesion in traumatic anuria. J. Pathol. Bacteriol. 54:111-120.

31. Lucke, B. 1946. Lower nephron nephrosis. Milit. Surg. 99:371396.

32. Oliver, J., M. McDowell, and A. Tracy. 1951. The pathogenesis of acute renal failure associated with traumatic and toxic injury. Renal ischemia, nephrotoxic damage and the ischemuric episode. J. Clin. Invest. 30:1307-1351.

33. Sevitt, S. 1956. Distal tubular necrosis with little or no oliguria. J. Clin. Pathol. (Lond.). 9:12-30.

34. Sevitt, S. 1959. Pathogenesis of traumatic uraemia. A revised concept. Lancet. II:135-141.

35. Kramer, H. J., J. Schüürmann, C. Wasserman, and R. Dusiney. 1980. Prostaglandin-independent protection by furosemide from oliguric ischemic renal failure in conscious rats. Kidney Int. 17:455-464.

36. Vatner, S. F. 1974. Effects of hemorrhage on regional blood flow distribution in dogs and primates. J. Clin. Invest. 54:225-235.

37. Eknoyan, G., W. Y. Quinibi, R. T. Grissan, S. N. Tuma, and J. C. Ayus. 1982. Renal papillary necrosis. An update. Medicine (Baltimore). 61:55-73. 